\title{
It's not all about the size-characteristics and risk factors for malignancy of mucinous cystic neoplasms of the pancreas
}

\author{
Philipp Höhn ${ }^{1}$, Muhammed Abdussamed Soydemir ${ }^{2}$, Andreas Minh Luu ${ }^{1}$, Monika Janot-Matuschek ${ }^{1}$, \\ Andrea Tannapfel ${ }^{3}$, Waldemar Uhl $^{1}$, Orlin Belyaev ${ }^{1}$ \\ ${ }^{1}$ Department of General and Visceral Surgery, St. Josef-Hospital, Ruhr-University Bochum, Bochum, Germany; ${ }^{2}$ Faculty of medicine, Ruhr- \\ University Bochum, Bochum, Germany; ${ }^{3}$ Institute of Pathology, Ruhr-University Bochum, Bochum, Germany \\ Contributions: (I) Conception and design: O Belyaev, W Uhl; (II) Administrative support: A Tannapfel, W Uhl; (III) Provision of study materials \\ or patients: M Janot-Matuschek, MA Soydemir, A Tannapfel; (IV) Collection and assembly of data: MA Soydemir, P Höhn; (V) Data analysis and \\ interpretation: P Höhn, O Belyaev; (VI) Manuscript writing: All authors; (VII) Final approval of manuscript: All authors. \\ Correspondence to: Philipp Höhn. Gudrunstr. 56, 44791 Bochum, Germany. Email: philipp.hoehn@rub.de.
}

Background: Mucinous cystic neoplasms (MCN) of the pancreas are rare mucin-producing cystic tumors. As they harbor malignant potential, surgical resection is frequently performed. Current guidelines recommend surgery in asymptomatic patients only for MCN exceeding $4 \mathrm{~cm}$. The aim of this study was to identify radiological and clinical risk factors for malignancy in a single-center cohort of MCN.

Methods: All resected MCN from a single high-volume center between 2004 and 2019 were retrospectively analyzed. Patient characteristics, preoperative findings, histopathological results, and data on the postoperative course were recorded. Variables associated with malignancy were evaluated using $\chi^{2}$ and Mann-Whitney U test. Receiver operating characteristic (ROC) curves were used to model predictive capabilities of preoperative tumor marker levels. Furthermore uni- and multivariate logistic regression analysis were performed for binary variables. Survival time was plotted as Kaplan-Meier curves and evaluated by log-rank test.

Results: A total of 63 patients were included. Median age was 62 years; 51 (81.0\%) of them were women; median tumor size was $3.5 \mathrm{~cm}$ (range, $0.5-18.5)$; 16 (25.4\%) of tumors harbored invasive carcinoma and 13 presented intraepithelial dysplasia (20.6\%); 7 (43.8\%) invasive carcinomas were smaller than $4 \mathrm{~cm}$. All malignant MCN were radiologically suspected of malignancy (calcifications, mural nodules, or wall thickness) preoperatively. Elevated levels of carbohydrate antigen 19-9 (CA19-9) and carcinoembryonic antigen (CEA) were strongly associated with malignancy (odd's ratio 33.600; 7.000-161.270); $\mathrm{P}<0.001$ and odd's ratio $19.250 ; 3.370-109.970 ; \mathrm{P}<0.001)$. Other factors associated with malignancy were preoperative weight loss $(\mathrm{P}=0.015)$ and higher age $(\mathrm{P}=0.048)$. Tumor size, abdominal or back pain or jaundice showed no significant correlation to malignancy in our cohort.

Conclusions: Malignant potential of MCN should not be underestimated and a close clinical and radiological follow-up is mandatory in all suspected cases. This is especially important for small lesions. Risk assessment should not rely only on tumor size but consider all clinical, radiological and laboratory findings of each case. Follow-up should be performed by experienced surgeons and radiologists in high volume centers for pancreatic surgery. Surgery should be performed in all cases in which malignancy is suspected.

Keywords: Mucinous cystic neoplasm (MCN); risk of malignancy; retrospective study

Submitted Jun 17, 2020. Accepted for publication Sep 25, 2020.

doi: 10.21037/atm-20-4774

View this article at: http://dx.doi.org/10.21037/atm-20-4774 


\section{Introduction}

Mucinous cystic neoplasms (MCN) belong to the neoplastic cystic tumors of the pancreas. Often benign, they have the potential to evolve into invasive cancer. Predominantly found in middle-aged women, they are usually located in the pancreatic tail or body (Figure 1). The presence of ovarian-type stroma differentiates them from other mucin producing neoplasms (1). MCN have been classified as a separate entity from intraductal papillary mucinous neoplasms (IPMN) by the World Health Organization (WHO) since 1996 (2). Discussion about the management of MCN has mainly revolved around the questions in which cases surgical resection is advised and how followup for non-resected patients should be conducted. In recent years, three major guidelines regarding this topic have been published (3-5). In case of asymptomatic tumors without other radiological signs of malignancy (calcifications, mural nodules or wall thickness) a size-threshold of $4 \mathrm{~cm}$ was proposed. For smaller tumors a watch-and-wait management is currently favored over surgery.

The aim of this study was to test the validity of this recommendation in a cohort of patients with resected $\mathrm{MCN}$ in a high-volume center for pancreatic surgery. Also, we aimed to identify clinical, radiological and laboratory features associated with malignancy in MCN. We present the following article in accordance with the STROBE reporting checklist (available at http://dx.doi.org/10.21037/atm-20-4774).

\section{Methods}

\section{Data acquisition}

We searched our in-house patient database for all patients who underwent pancreatic resection for MCN between 2004 to 2019. Exclusion criteria were missing consent and/ or incomplete documentation. Histopathological findings were reviewed to ensure presence of ovarian-like stroma in all patients. Written consent was obtained from all patients. The study was approved by the local ethics committee (Nr. 17-6161). The study was conducted in accordance with the Declaration of Helsinki (as revised in 2013).

\section{Parameters}

Detailed perioperative characteristics were extracted for every patient, including laboratory, radiological, histopathological and intraoperative findings, as well as data regarding the postoperative course (Tables 1,2).
POPF was defined according to the guidelines by the International Study Group on Pancreatic Fistulas (ISGPF) (6). Complications were sorted according to the Clavien-Dindo classification (7). Mortality was defined as mortality within 30 days of surgery independent of hospitalization status. Symptoms were classified as related if abdominal or back pain were present and couldn't be attributed to other diseases in the patient's history. Weight loss was defined as decrease of $\geq 10 \%$ of bodyweight in 12 months. Exocrine insufficiency was defined as preoperative stool elastase $<200 \mu \mathrm{g} / \mathrm{g}$ and/or clinical symptoms of malabsorption. Radiological signs of malignancy were defined as wall thickness (cyst wall $>3 \mathrm{~mm}$ ), calcifications and mural nodules. Dilatation of the pancreatic duct $>3 \mathrm{~mm}$ was not considered a radiological sign of malignancy.

\section{Statistical analysis}

Comparison between group demographic data was performed by Student's unpaired $t$-test for normally distributed continuous variables and the Mann-Whitney $\mathrm{U}$ test for non-normally distributed variables. Receiver operating characteristic (ROC) curves were used to model predictive capabilities of preoperative tumor marker levels. Goodness of fit was assessed by calculating the areas under the curve (AUC). $\chi^{2}$-test was used for categorical data and Fisher's exact test for cell counts lower than 5. Patients with missing data for respective variables were excluded from statistical analysis. Univariate analysis was performed to identify risk factors that correlated with the presence of malignancy. To identify independent risk factors, binary logistic regression analysis was performed. Parameters for multivariate analysis were selected by a $\mathrm{P}$ value $<0.05$ after univariate analysis. Variables were entered simultaneously into the regression model. Laboratory findings and clinical symptoms were analyzed separately $(8,9)$. Patient survival was evaluated using Kaplan-Meier curves and log-rank test. Patients lost during follow-up were censored and not included in survival time analysis. Tests were designed 2 -tailed with the statistical significance level set at $\mathrm{P}<0.05$. All analyses were performed with SPSS version 26.0 software (IBM, Armonk, New York, NY).

\section{Results}

\section{Patient population and radiographic features}

If not indicated otherwise, results are presented in absolute 

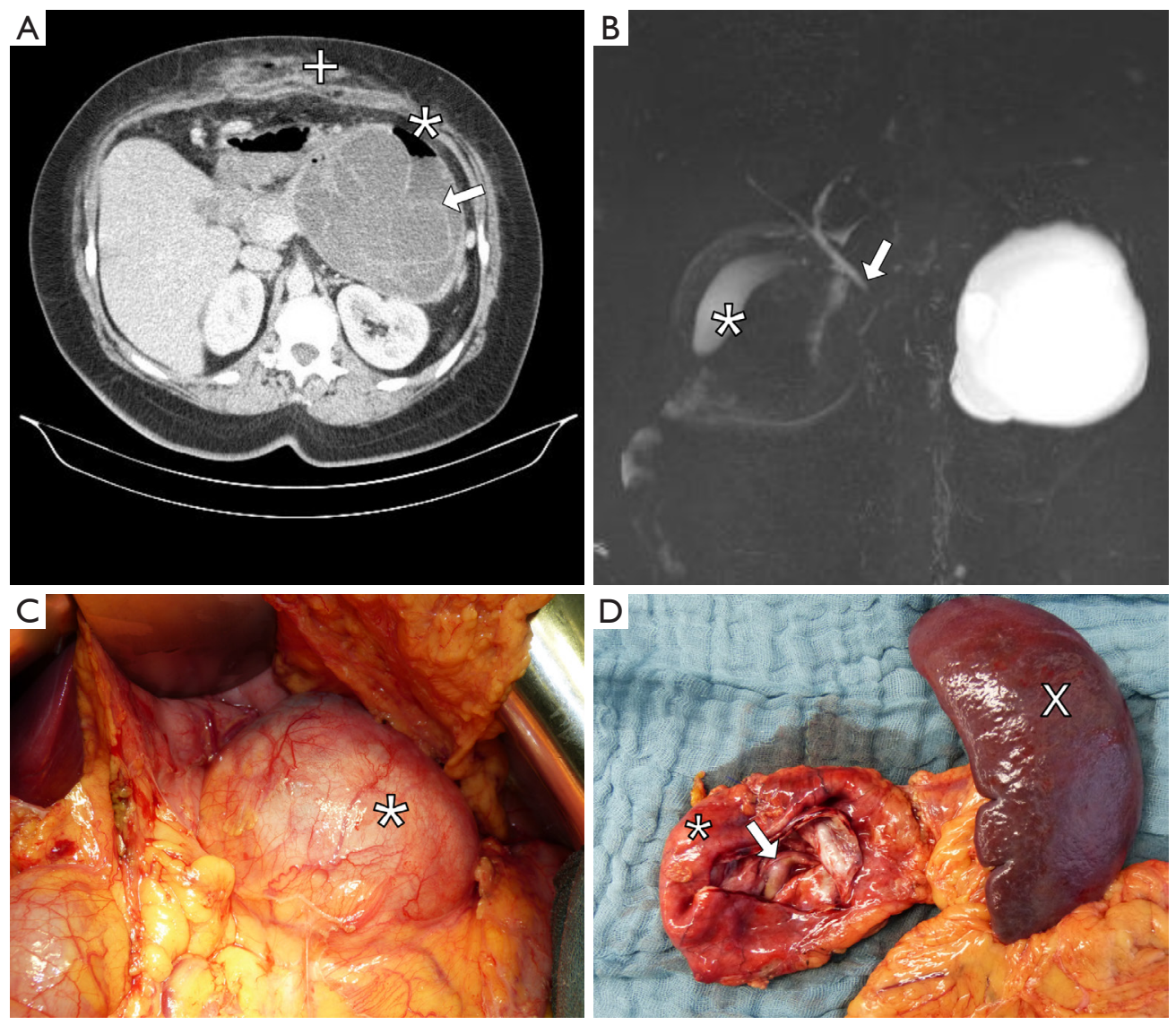

Figure 1 Radiographic and macroscopic features of MCN. (A) A computed tomography scan of the upper abdomen including a large septated cystic mass at the pancreatic tail (arrow). Intracystic air (asterisk) and subcutaneous soft tissue enhancement (cross) are remnants of an external unsuccessful attempt of cyst deroofing. (B) A magnetic resonance cholangiopancreatography showing a large cystic mass in the left upper abdomen. Also visible are gallbladder (asterisk) and common bile duct (arrow). (C) Macroscopic aspect of a large MCN (asterisk) before distal pancreatectomy. (D) The specimen after distal pancreatectomy, including the spleen (X). The thick wall of the MCN (asterisk) and intracystic septs (arrow) are apparent.

numbers followed by percentage in brackets or as median values followed by range in brackets.

In total, 63 patients were included in our study. All of them underwent resection for MCN between 2004 and 2019. The number of cases per year ranged from 1 to 13 with an average of 4.2 .

The sex ratio was $4.3: 1$ for women to men. The range of age was 56 years (29-85 years); 4 of 16 malignant MCN occurred in men (25\%); 46 patients (71.9\%) showed MCN related symptoms. The vast majority of symptomatic patients complained of abdominal or back pain (91.5\%). $4.8 \%$ of patients with MCN presented with jaundice. Jaundice was not associated with a tumor location in the pancreatic head $(\mathrm{P}=0.272)$. Preoperative diabetes mellitus was more common than exocrine pancreatic insufficiency
(65.1\% vs. 14.3\%). Also, exocrine pancreatic insufficiency was associated with a tumor location in the pancreatic head $(\mathrm{P}=0.037) .57 .2 \%$ of our population were at least overweight. 26 patients $\left(41.3 \%\right.$ ) had a BMI $>25 \mathrm{~kg} / \mathrm{m}^{2}$ and 10 patients (15.9\%) presented with a BMI $>30 \mathrm{~kg} / \mathrm{m}^{2}$.

Thirty-eight $(60.3 \%) \mathrm{MCN}$ were located in the pancreatic tail and $21(33.4 \%)$ were located in the pancreatic head. Tumor location in the pancreatic head was strongly associated with male sex $(\mathrm{P}<0.001)$ but not with malignancy $(\mathrm{P}=1.000)$. Median tumor size was $35 \mathrm{~mm}$ (range, $5-185 \mathrm{~mm}$ ). Mean tumor size was $44.09 \mathrm{~mm}$ [standard deviation (SD): 29.43] for benign MCN and $52.29 \mathrm{~mm}$ (SD: 44.75) for malignant cases. All malignant MCN had radiological features of malignancy (calcifications, mural nodules, or wall thickness); 26 tumors (41.3\%) 
Table 1 Characteristics, clinical and radiological findings of study cohort $(n=63)$

\begin{tabular}{|c|c|}
\hline Characteristics & Values \\
\hline \multicolumn{2}{|l|}{ Demographics } \\
\hline Sex ratio (F:M) & $51 / 12$ \\
\hline Age (years) & $62(29 \text { to } 85)^{*}$ \\
\hline $\operatorname{BMI}\left(\mathrm{kg} / \mathrm{m}^{2}\right)$ & $25(16 \text { to } 42)^{\star}$ \\
\hline $25-30$ kg/m², n (\%) & $26(41.3)$ \\
\hline$>30$ kg/m², n (\%) & $10(15.9)$ \\
\hline \multicolumn{2}{|l|}{ Presence of symptoms, n (\%) } \\
\hline Pain & $43(68.3)$ \\
\hline Weight loss & $16(25.4)$ \\
\hline Jaundice & $3(4.8)$ \\
\hline \multicolumn{2}{|l|}{ Medical history, n (\%) } \\
\hline Diabetes & $41(65.1)$ \\
\hline Exocrine insufficiency & $9(14.3)$ \\
\hline Family history & $2(3.2)$ \\
\hline Nicotine consumption & $10(15.9)$ \\
\hline Alcohol consumption & $7(11.1)$ \\
\hline \multicolumn{2}{|l|}{ ASA Score, n (\%) } \\
\hline 1 & $3(4.8)$ \\
\hline 2 & $30(47.6)$ \\
\hline 3 & $27(42.9)$ \\
\hline 4 & $1(1.6)$ \\
\hline \multicolumn{2}{|l|}{ Radiological findings } \\
\hline Tumor size $(\mathrm{mm})$ & $35(5 \text { to } 185)^{\star}$ \\
\hline Size >4 cm, n (\%) & $26(41.3)$ \\
\hline \multicolumn{2}{|l|}{ Tumor location, $\mathrm{n}(\%)$} \\
\hline Head & $21(33.4)$ \\
\hline Body & $4(6.3)$ \\
\hline Tail & $38(60.3)$ \\
\hline \multicolumn{2}{|l|}{ Histopathological findings, $\mathrm{n}(\%)$} \\
\hline Invasive carcinoma & $16(25.4)$ \\
\hline High grade intraepithelial dysplasia & $0(0.0)$ \\
\hline Low grade intraepithelial dysplasia & $13(20.6)$ \\
\hline Adenoma without dysplasia & $34(54.0)$ \\
\hline Lymph node involvement & $5(31.3)^{\star \star}$ \\
\hline Metastasis & $5(31.3)^{\star \star}$ \\
\hline Complete resection & $56(88.8)$ \\
\hline
\end{tabular}

Table 1 (continued)
Table 1 (continued)

\begin{tabular}{|c|c|}
\hline Characteristics & Values \\
\hline \multicolumn{2}{|l|}{ Type of surgery, n (\%) } \\
\hline Pancreaticoduodenectomy & $15(23.8)$ \\
\hline \multicolumn{2}{|l|}{ Distal pancreatectomy } \\
\hline With splenectomy & $10(15.9)$ \\
\hline Without splenectomy & $27(42.2)$ \\
\hline \multicolumn{2}{|l|}{ Total pancreatectomy } \\
\hline With splenectomy & $1(1.6)$ \\
\hline Without splenectomy & $5(7.9)$ \\
\hline Other & $5(7.9)$ \\
\hline Multivisceral resection & $9(14.3)$ \\
\hline \multicolumn{2}{|l|}{ Outcome, n (\%) } \\
\hline Perioperative 30 -day mortality & $5(7.9)$ \\
\hline 30-day intervention & $18(28.6)$ \\
\hline Postoperative pancreatic fistula & $20(31.7)$ \\
\hline Biochemical leak & $6(9.5)$ \\
\hline $\mathrm{B}$ & $20(31.7)$ \\
\hline $\mathrm{C}$ & $0(0.0)$ \\
\hline \multicolumn{2}{|l|}{ Clavien Dindo grading, $\mathrm{n}(\%)$} \\
\hline I & $6(9.5)$ \\
\hline II & $31(49.2)$ \\
\hline III & $11(17.5)$ \\
\hline IV & $2(3.2)$ \\
\hline V & $5(7.9)$ \\
\hline
\end{tabular}

Percentages are relative to all 63 cases unless indicated otherwise. ${ }^{*}$, values are median (range); ${ }^{* *}$, percentages are relative to the number of invasive cancer cases $(n=16)$.

were larger than $4 \mathrm{~cm}$. However, a tumor size $>4 \mathrm{~cm}$ did not correlate with malignancy $(\mathrm{P}=0.159)$ and ranked distribution of tumor size was indifferent between malignant and benign MCN $(\mathrm{P}=0.778)$. Five of the 16 carcinoma were smaller than $2 \mathrm{~cm}(31.3 \%)$.

\section{Histopathological results}

Sixteen $(25.4 \%)$ of 63 tumors contained invasive adenocarcinoma. $31.3 \%$ (5 of 16 ) of invasive tumors presented with lymph node involvement or intraabdominal metastasis. 3 of 16 tumors showed both (18.8\%). Only 1 T1 carcinoma was found (6.3\%). 3 tumors were classified 
Table 2 Comparison of continuous parameters

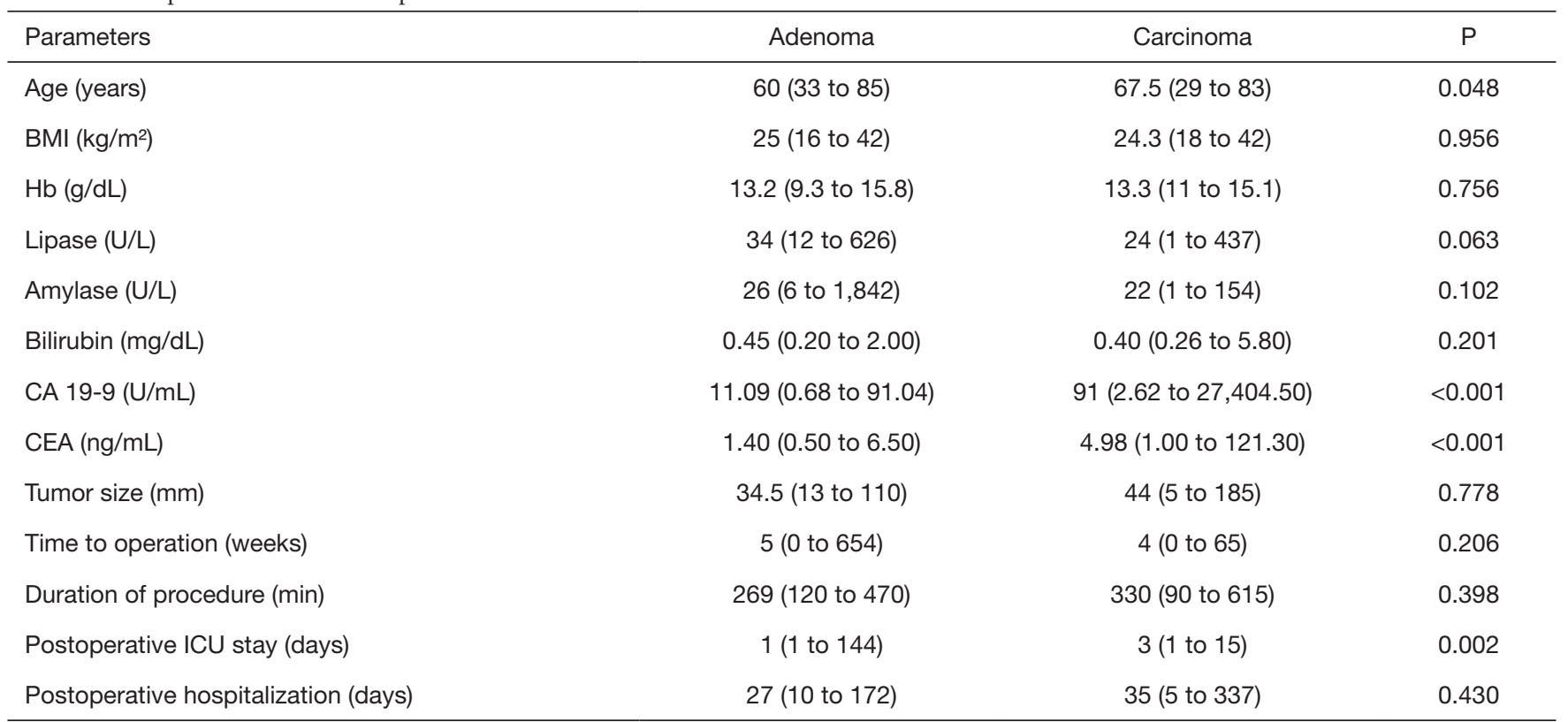

Distribution of continuous parameters for different histological results compared by Mann-Whitney $U$ test. All values are presented as median (range). Laboratory findings are preoperative serum levels. Time to operation refers to the time between first recorded onset of abdominal symptoms and operation. HB, hemoglobin; CA19-9, carbohydrate antigen 19-9; CEA, carcinoembryonic antigen.

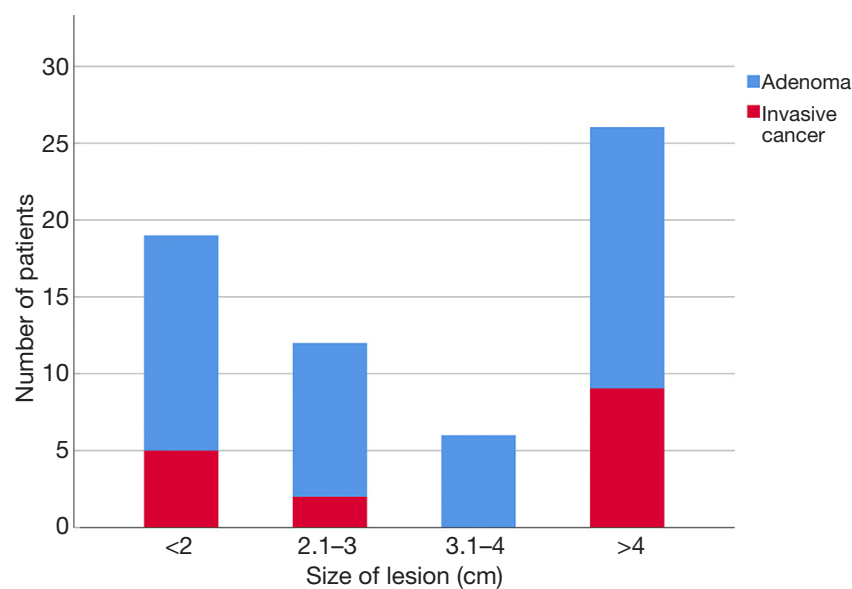

Figure 2 Distribution of MCN being adenomas or invasive cancer (IC) sorted by the lesions' size. Smaller than $2 \mathrm{~cm}$ : $5 \mathrm{IC}$ and 14 adenomas; $2.1-3 \mathrm{~cm}: 2$ IC and 10 adenomas; $3.1-4 \mathrm{~cm}$ : 6 adenomas; bigger than $4 \mathrm{~cm}$ : $9 \mathrm{IC}$ and 17 adenomas.

T2 $(18.8 \%)$ and 6 tumors were classified T3 and 5 tumors as T4 (37.5\% and $31.3 \%)$. For 1 tumor $(6.3 \%)$ a T stadium could not be obtained. 3 of all MCN in which curative resection was attempted were incompletely resected (5.1\%), all of them being carcinomas. Curative resection rate for malignant disease was $75 \%$ ( 9 of 12). Grading was G1 in 6 tumors (37.5\%), G2 and G3 was found in 5 tumors each (both $31.3 \%$ ). Further data on clinical, histological, and radiological characteristics of all patients are shown in Table 1. Figure 2 presents the distribution of adenomas versus invasive cancer for categorized tumor sizes.

\section{Procedures and outcome}

Distal pancreatectomy was performed in 37 patients (58.8\%). $27.0 \%$ of these cases included simultaneous splenectomy (10 of 37 ). Performed in 15 patients $(23.8 \%)$, the second most common procedure was pancreaticoduodenectomy. In 9 cases $(14.3 \%)$ a multivisceral resection was conducted. This included partial resection of liver, suprarenal gland, stomach, or colon. Six of 9 multivisceral resections were performed for benign MCN (66.7\%).

$22.8 \%$ of all patients developed a clinically relevant POPF after partial pancreatic resection (13 of 64). Fistula rate after distal pancreatectomy was $35.1 \%$ (13 of 37 ). Thirteen of all partial pancreatic resections presented a biochemical leak (22.8\%). No grade C fistula was observed. Postoperative mortality was $7.9 \%$ (5 of 63 patients) and 


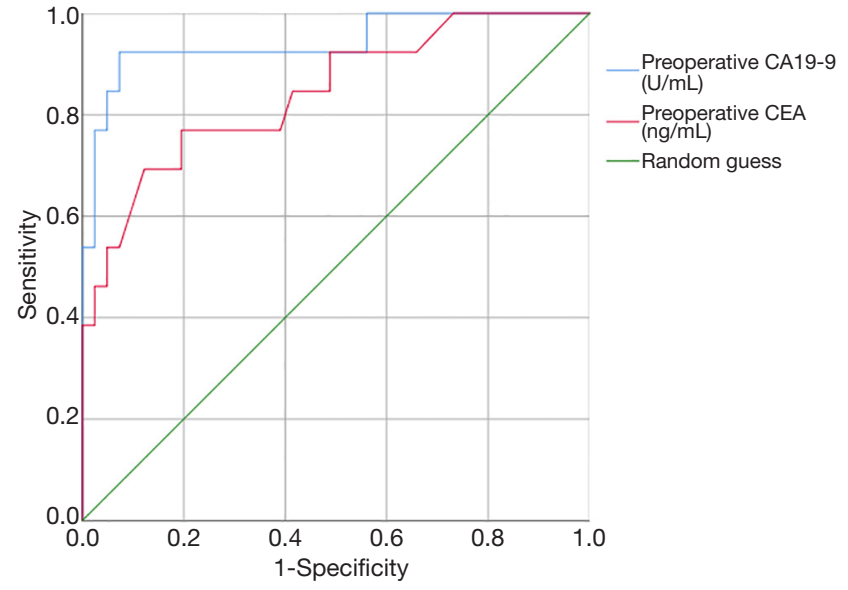

Figure 3 Receiver operating characteristic curves for preoperative CEA and CA19-9 levels as predictors of malignancy in MCN. AUC for CA19-9 is 0.942 and AUC for CEA is 0.842 .

Table 3 Comparison of categorical parameters

\begin{tabular}{lccc}
\hline Parameters & Adenoma & Carcinoma & $P$ \\
\hline Pain & 30 & 13 & 0.087 \\
Weight loss & 9 & 7 & $0.01^{*}$ \\
Jaundice & 1 & 2 & 0.099 \\
Diabetes & 30 & 10 & 0.754 \\
Exocrine insufficiency & 4 & 5 & 0.039 \\
Family history & 2 & 0 & 1.000 \\
Nicotine consumption & 9 & 1 & 0.650 \\
Alcohol consumption & 6 & 1 & 1.000 \\
Death & 0 & 5 & $<0.001$ \\
Size $>4$ cm & 17 & 9 & $0.159^{\star}$ \\
Anemia & 5 & 2 & 1.000 \\
BMl $>25 \mathrm{~kg} / \mathrm{m}^{2}$ & 19 & 8 & 0.770 \\
BMl $>30 \mathrm{~kg} / \mathrm{m}^{2}$ & 7 & 4 & 0.696 \\
Male sex & 4 & 8 & 0.718 \\
Pancreatic head & 5 & 6 & 1.000 \\
Elevated CA19-9 & 5 & 7 & $<0.001$ \\
Elevated CEA & 5.001 \\
\hline
\end{tabular}

Frequencies of categorial parameters for different histological results compared by Fisher's exact test. $n=16$ for carcinomas and $n=47$ for adenomas. *, the use of Chi-Square test. evenly distributed among different procedures. $28.6 \%$ (18 of 63) of patients developed complications requiring intervention. $67.3 \%$ of complications were classified grade 1 or 2 according to Clavien-Dindo classification (37 of 55). The next most common grade was $3 \mathrm{a}$, including 9 complications (16.7\%).

Overall median length of postoperative hospitalization was 27 days and did not differ significantly between malignant or benign cases $(\mathrm{P}=0.687)$. However, median postoperative ICU admission was significantly longer for patients with malignant disease ( 3 vs. 1 days; $\mathrm{P}=0.002$ ). Median duration of procedures was 270 minutes with a range of 90 to 615 minutes. On average, procedures for malignant MCN did take longer, but the difference was not significant $(\mathrm{P}=0.398)$. Median time between established diagnosis of suspect pancreatic cyst and operation was 5 weeks.

\section{Parameters associated with malignancy}

Weight loss was the only clinical finding that was significantly associated with the presence of malignancy $(\mathrm{P}=0.01)$. Regarding laboratory findings, median lipase levels were lower in patients with malignant MCN. Median age was higher in patients with carcinomas. The difference was statistically significant but presented a high $\mathrm{P}$ value $(\mathrm{P}=0.046)$. Highly significant differences $(\mathrm{P}<0.001)$ between malignant and benign cases were observed for elevated CA19-9 (>27 U/mL) and CEA levels ( $>5 \mathrm{ng} / \mathrm{mL})$. Goodness of fit for a model using tumor marker levels as a predictor for malignancy resulted in an area under the curve (AUC) of 0.942 for CA19-9, indicating an excellent fit, and an AUC of 0.842 for CEA, indicating a good fit. Routine laboratory thresholds for pathological values as described above demonstrated a sensitivity of $86.7 \%$ and specificity of $88.1 \%$ for CA19-9 and a sensitivity of $50 \%$ and specificity of $97.8 \%$ for CEA (Figure 3).

Binary logistic regression analysis confirmed weight loss, elevated CA19-9 and elevated CEA levels to be associated with malignant disease. Another clinical finding that showed a weaker association with malignancy was exocrine pancreatic insufficiency $(\mathrm{P}=0.034)$. Weight loss and elevated CA19-9 levels were also significant independent risk factors in multivariate analysis. Further results and $\mathrm{P}$ values are shown in Tables 2-4. 
Table 4 Logistic regression analysis of potential risk factors for malignancy

\begin{tabular}{|c|c|c|c|c|}
\hline Risk factors & Univariate analysis & $\mathrm{P}$ & Multivariate analysis & $P$ \\
\hline Pancreatic insufficiency & 5.357 (1.136-25.264) & 0.034 & - & - \\
\hline Elevated CA19-9 & $33.600(7.000-161.270)$ & $<0.001$ & $21.620(4.168-112.136)$ & $<0.001$ \\
\hline Elevated CEA & $19.250(3.370-109.970)$ & $<0.001$ & $7.778(0.905-66.875)$ & 0.062 \\
\hline
\end{tabular}

Uni- and multivariate binary logistic regression analysis for categorical variables. Pancreatic insufficiency refers to exocrine pancreatic insufficiency. Values are odd's ratio $(95 \% \mathrm{Cl})$.

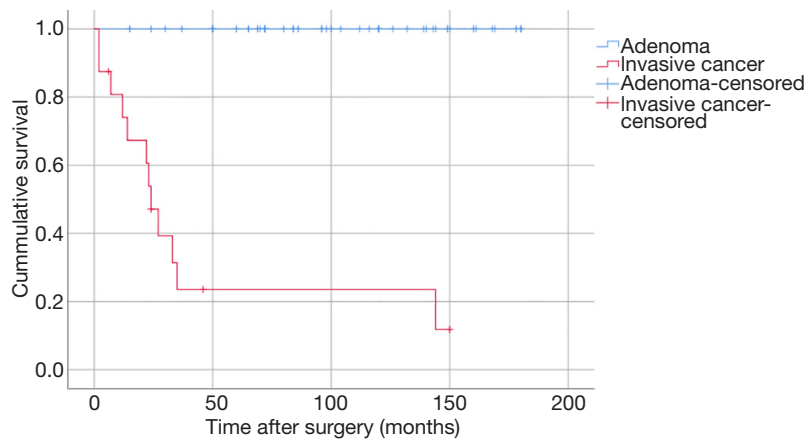

Figure 4 Kaplan-Meier survival curves for patients with adenoma or invasive cancer. $\mathrm{P}<0.001$ (log-rank test).

\section{Follow-up, survival, and recurrence}

Overall follow-up ranged between 2 to 180 months (mean: 87 months, SD: 55 months). Follow-up on patients with adenoma was completed in 33 cases $(70.2 \%)$. Follow-up time ranged from 15 to 180 months (mean: 100 months, SD: 46 months). No patient with non-invasive disease died during the follow-up period and no recurrence of $\mathrm{MCN}$ was observed.

Regarding patients with invasive cancer, follow-up was completed in 13 cases $(81.2 \%)$. Follow-up time ranged from 2 to 150 months (mean: 36 months, SD: 45 months). Follow-up on one Patient with invasive cancer was lost after 6 months. 12 patients $(75 \%)$ with invasive disease died during the follow-up period. Median survival time was 24 months. Five-year survival rate was $23.6 \%$. Figure 4 shows Kaplan-Meier curves for survival stratified by presence of invasive cancer. Log-rank test for uneven survival between groups was highly significant $(\mathrm{P}<0.001)$.

None of the patients with benign MCN developed any recurrence of MCN during follow-up. However, one male patient underwent distal pancreatectomy in 2005 for benign MCN adenoma and then underwent completion pancreatectomy in 2019 because of pancreatic ductal adenocarcinoma in the region of the pancreatic stump. The patient received adjuvant chemotherapy and is still alive 180 months after diagnosis of $\mathrm{MCN}$ and 16 months after diagnosis of ductal adenocarcinoma. Another female patient with distal pancreatectomy for benign MCN developed ovarian cancer 60 months later, but after successful treatment is currently still alive at 100 months follow-up.

All patients with lethal outcome after surgery for invasive malignant MCN died of diffuse metastatic disease, apart from the one who died of a stroke 2 months after surgery. There was only one recorded local recurrence in a female patient who underwent total pancreatectomy for a large malignant MCN. Despite refusing adjuvant chemotherapy she lived disease-free for 60 months after surgery before a local recurrence at the VMS was detected and then surgically removed. Numerous surgical procedures followed with removal of different localized metastases and the patient died 144 months after initial surgery. Table 5 summarizes follow-up, survival, and recurrence data.

\section{Discussion}

\section{Current guidelines and treatment recommendations}

MCN of the pancreas were first differentiated from serous cystic neoplasms in 1978 as cystic neoplasms forming papillae lined with columnar, mucin-producing epithelium (1). They were recognized as an independent entity and distinguished from intraductal papillary mucinous neoplasms of the pancreas (IPMN) by the World Health Organization (WHO) in 1996. Since 2000 the WHO's definition emphasizes the existence of ovarianlike stroma to differentiate between MCN and other cystic neoplasm of the pancreas. In contrast to previous editions, the current WHO definition classifies lesions including a carcinoma in situ as (still) benign tumors (10). MCN occur 
Table 5 Follow-up, survival, and recurrence

\begin{tabular}{lcc}
\hline Parameter & Adenoma & Carcinoma \\
\hline Follow up quality & $33(70.2)$ & $13(81.2)$ \\
Complete, $\mathrm{n}(\%)$ & $14(29.8)$ & $3(18.2)$ \\
Lost, $\mathrm{n}(\%)$ & $87(55.0)$ & $36(45.0)$ \\
Follow up time (months) & & \\
Survival & $33(100.0)$ & $1(7.7)$ \\
Alive, $\mathrm{n}(\%)$ & $0(0.0)$ & $12(92.3)$ \\
Dead, $\mathrm{n}(\%)$ & $\mathrm{NA}$ & $38.08(49.51)$ \\
Survival time (months), mean (SD) & $\mathrm{NA}$ & 23.6 \\
5-year survival rate (percent) & & \\
Recurrence, $\mathrm{n}$ (\%) & $0(0.0)$ & $11(84.6)$ \\
Recurrence of MCN & $\mathrm{NA}$ & $1(9.1)$ \\
Local recurrence & $\mathrm{NA}$ & $10(90.9)$ \\
Distant recurrence & $2(6.1)$ & $0(0.0)$ \\
Other metachronous carcinoma & $0(0.0)$ & $1(7.7)$ \\
Non-MCN related death & & \\
\hline
\end{tabular}

Values are presented as absolute numbers, followed by percentage values in brackets unless indicated otherwise. Percentages for survival and recurrence are based on 33 patients. NA, not applicable.

predominantly in women (female: male ratio 20:1). Peak incidence is in the 5 th decade and they're mainly found in the pancreatic body or tail (93-95\%). Communication with main pancreatic duct is very rarely observed and normally attributed to secondary fistula formation. Reported size of $\mathrm{MCN}$ range from 2-36 cm with an average of 7-10 cm (11).

Discussion about management of MCN has mainly revolved around the questions in which cases surgical resection is advised and how follow up for non-resected patients should be conducted. In recent years, three major guidelines regarding this topic have been published. The international consensus guidelines published by Tanaka et al. in 2012 did recommend surgical resection for any confirmed MCN in surgically fit patients. For lesions $<4 \mathrm{~cm}$ and without mural nodules, parenchyma-sparing and, if possible, laparoscopic resections should be considered. Pancreatectomy with lymph node dissection remains the standard treatment for invasive and non-invasive MCNs (4). The guideline's revision in 2017 explicitly excluded MCN stating that "there are very few remaining points of controversy regarding this entity" (12). The 2015 guideline published by the American Gastroenterological Association Institute recommends endosonographic ultrasound (EUS) and fine-needle aspiration (FNA) for all cysts with at least 2 high-risk features (size $\geq 3 \mathrm{~cm}$, presence of an associated solid component or dilated main pancreatic duct). Surgery is recommended for all patients with worrisome features in imaging and/or suspicious cytology findings after FNA. Initial evaluation by magnetic resonance imaging (MRI) for unsuspicious cysts $<3 \mathrm{~cm}$ is recommended. Followup should be performed after 1 year and afterwards every 2 years for a total period of 5 years (5). Most recent the European Study Group on Cystic Tumors of the Pancreas published European guidelines on pancreatic cystic neoplasms. Resection is recommended for MCN $>4 \mathrm{~cm}$ or if radiological risk factors are present (i.e., mural nodules). For lesions between 3 and $4 \mathrm{~cm}$, other factors (age, comorbidities, and patient preference) should be considered. Surveillance is recommended for lesions $<3 \mathrm{~cm}$ similar to IPMN. Follow up for MCN $<4 \mathrm{~cm}$ is recommended by MRI or EUS initially every 6 months and once a year afterwards. EUS is only recommended if results are expected to change clinical management (3).

All three guidelines consent that surgery for cystic pancreatic neoplasm should be performed in high volume centers and that resection is recommended for all symptomatic pancreatic cysts. Furthermore, evidence of most recommendations is considered low as data is derived mainly from retrospective analysis of resected patients (3-5).

\section{Challenges in preoperative determination of malignancy}

Correct preoperative diagnosis of cystic pancreatic lesions remains challenging. Retrospective analysis of 141 pancreatic resections in a high-volume tertiary referral center revealed a diagnostic accuracy of $72 \%$ for $\mathrm{MCN}$ after assessment by CT, MRI or EUS + FNA. The majority of patients received more than one diagnostic procedure (13). Another similar study, including 174 patients, found an even lower accuracy for MCN of 54\% which was also significantly lower than in other pancreatic cysts. Correctly characterized $\mathrm{MCN}$ were significantly bigger than incorrect characterized MCN (14). Our data did not show a significant association between a tumor location in the pancreatic head and malignancy in MCN. We could only find one study demonstrating a correlation between tumor location and malignancy (15). This finding was not replicated by later studies (11). Studies focusing on the size of cystic neoplasms as a predictor of malignancy report a specificity 
as low as $46 \%$ for a threshold of $4.5 \mathrm{~cm}$ (16). In contrast, radiological findings of calcifications, wall thickness and septations can strongly increase the probability of correct radiological diagnosis. These attributes also correlate with the presence of malignancy with an estimated probability of $95 \%$ if all three are present (15). Our study could not find a correlation between MCNs' size and malignancy. Median tumor size did not differ between groups. Malignancy did not occur more often in lesions bigger than $4 \mathrm{~cm}$. Five of 16 carcinomas $(31.3 \%)$ were smaller than $2 \mathrm{~cm}$. We find it important to stress that malignancy in MCN may occur independent of the lesions' size.

Cystic pancreatic lesions are long known to harbor malignant potential (1). Data on frequency of invasive carcinoma in MCN is primarily based on retrospective reports on resected tumors. Reported malignancy rates changed over the last decades as more precise definitions by the WHO resulted in a stricter separation between IPMN and MCN (10). Studies focusing on MCN defined by ovarian-like stroma report higher malignancy rates than those with other diagnostic criteria (17). Including carcinoma in situ, studies report malignancy rates of up to $54 \%$ (18). Considering only invasive carcinoma, two studies with a total of 88 patients reported rates of $29-34 \%(19,20)$. Average rates of malignancy and tumor size gradually declined in recent years as large retrospective studies reported lower incidence of $\mathrm{CiS}$ and invasive carcinoma (17\% and $12-13 \%)(17,21)$. Our study found $26.6 \%$ of MCN harboring invasive carcinoma. This number is higher than in recent multicenter studies. It could be attributed either to an unknown geographic difference in prevalence or to a more efficient selection of patients for surgery. In our clinic indication for surgery was confirmed by experienced pancreatic surgeons and strictly tied to high suspicion of malignancy. Criteria suggesting malignancy were continuously applied throughout the whole study period. This could, at least partially, explain a higher portion of malignant $\mathrm{MCN}$ in our collective.

\section{Etiological and clinical findings associated with malignancy}

In previous studies etiological factors associated with malignant disease were higher age, male sex, and high body mass index $(22,23)$. Our cohort included a relatively high portion of men $(18.8 \%)$ relative to other studies $(0-5 \%)(17,18)$. This is likely the reason why male sex was not significantly associated with malignancy in our study $(\mathrm{P}=0.718)$. Previous findings of more frequent occurrence of MCN located in the pancreatic head in male patients were replicated in our study. However, causal mechanisms still remain elusive. The high proportion of men in our study could be attributed either to an unknown geographic difference in prevalence or to a selection bias. Unfortunately, we cannot provide a definitive explanation for the high overall proportion of men in our study. Multiple studies found that symptoms like weight loss, back pain and jaundice in patients undergoing surgery for MCN were associated with malignancy $(23,24)$. In our study pain was more frequent in patients with invasive disease. However, difference did not reach statistical significance $(\mathrm{P}=0.085)$. Association of weight loss and invasive carcinoma has been shown in multiple studies before $(11,17,24)$. Correlation with malignancy was also present in our study $(\mathrm{P}=0.01)$. Also, we found weight loss to be an independent risk factor for malignancy in multivariate analysis. Involuntary weight loss is known to be associated with a multitude of malignant diseases (25). As clinical interpretation abdominal complaints might be heterogenous, we feel that weight loss might be a more objective finding.

\section{Role of serum tumor markers}

CA19-9 is the best validated serum tumor marker for pancreatic cancer (26). Recent studies on resected MCN found higher median serum levels in patients with invasive compared to non-invasive MCN. Also, elevation of serum levels beyond normal range was significantly more frequent in case of malignancy $(24,27,28)$. Both of these findings are strongly supported by our study. We found elevated CA19-9 levels to be an independent risk factor for malignant MCN after multivariate analysis. Elevation of CEA is more infrequent in patients with pancreatic cancer. Nevertheless, it is the most sensitive tumor marker for pancreatic cancer in patients with Lewis negative genotypes (29). Our study found elevated serum CEA levels to be a risk factor for malignant MCN. Other studies which evaluated serum CEA levels did not show similar associations $(22,27)$. Regarding the fact that most studies focus on CEA levels in cyst fluid, and very few report preoperative serum levels, we find it difficult to draw definitive conclusions on this point.

\section{Heterogenity of histological findings}

MCN frequently harbor different areas of adenoma including low and high grade dysplasia (LGD and HGD), even invasive carcinoma, in one lesion (30). 
Research by Izeradjene $e t$ al. showed that development of malignant $\mathrm{MCN}$ in mice is enacted by concomitant expression of KrasG12D and haploinsufficiency of the Smad4/Dpc4 tumor suppressor gene. Even though these genes also play a major role in the classical PanIN-toductal adeno- carcinoma sequence, genetic progression for MCN is markedly different (31). If this allows conclusion of an obligate adenoma to carcinoma sequence as observed in colonic cancer is currently debated $(4,19)$. Our study included 13 patients $(20.6 \%)$ in which low grade intraepithelial neoplasia was present but none with high grade intraepithelial dysplasia. These findings are nearly impossible to interpret, as reports of dysplasia in MCN are very heterogeneous. Some studies report HGD and carcinoma as a single group, whilst others group LGD with non-dysplastic adenoma $(17,19)$. The matter is further complicated by the terms carcinoma in situ and borderline tumors which are sometimes differently defined and/or grouped with HGD (11,32). We consider this a major weakness of available literature on MCN, making it very difficult to draw conclusions regarding the role of progressive dysplasia.

\section{Prognosis of MCN}

In terms of prognosis, complete resection is generally considered a curative treatment for benign $\mathrm{MCN}$ as reported recurrence rates range close to zero $(17,28)$. Presence of high-grade dysplasia or minimally invasive carcinoma are also associated with recurrence rates below $5 \%$ and 5 -year survival rates of $95-100 \%$. These findings are supported by our data, showing a 100\% 5-year survival rate for benign MCN. In contrast, prognosis is markedly worse once invasive carcinoma is present in MCN. Threeand 5 -year survival rates for invasive $\mathrm{MCN}$ are reported between $57 \%$ to $62.5 \%(27,28,33,34)$. In patients with invasive $\mathrm{MCN}, 25-71 \%$ suffer from tumor recurrence. $62.5 \%$ to $100 \%$ of these patients die of invasive cancer within 12 months after recurrence. Patients with lymph node involvement seem to be more at risk $(17,19,28,35)$. Our study found an even lower median survival and 5-year survival rate for invasive MCN than most other studies. This may be attributed to the low number of censored patients entering the survival analysis. Another possible factor influencing survival could be the higher average age of our collective compared to other studies. We believe that development of invasive carcinoma in $\mathrm{MCN}$ should be prevented early by aggressive surgical resection. This opinion is based on the fact that prognosis is markedly worse once malignancy is present in MCN.

\section{Strenghts, limitations and further studies}

Our study included a high number of consecutive patients from a single center in a relatively short timeframe of 15 years. We reported the highest number of malignant MCN with ovarian-like stroma from a single center in the current literature. Patients were assessed by CT and MRI scan and reviewed by the same team of experienced pancreatic surgeons over multiple years. Surgery could therefore not only be recommended by static criteria like tumor size, but also take into account dynamic changes in radiological or clinical findings. As no other way of definitive diagnosis is currently available, all data on $\mathrm{MCN}$ results from retrospective analysis of resected patients. Studies including more than 50 Patients and strict histopathological diagnosis criteria are almost all based on multicenter data. Furthermore, included patients often cover a long timeframe, easily exceeding 25 years or more $(17,18,21,30,33,36)$. To our knowledge, only two single-center studies mandating the presence of ovarian like stroma and including $>50$ patients exist. Reddy et al. reported a series of 56 patients over the course of 17 years from a single center in 2004 (30). Park et al. reported $90 \mathrm{MCN}$ from a single center who underwent surgery between 1991 to 2012, including 4 invasive carcinomas (27). Our analysis shares several limitations with the mentioned above studies. We only included patients who underwent surgery and performed a retrospective data analysis. This method can create strong selection bias which is difficult to compensate. Also, our study included only 17 MCN with associated carcinoma. This led to small sample sizes in subgroup analysis, resulting in large confidence intervals, further hampering statistical analysis.

Large prospective cohort studies of patients harboring cystic pancreatic lesions, ideally including multiple ethnic groups, could considerably increase our understanding of these entities. Unfortunately, this kind of studies might be nearly impossible to conduct, given the ever-looming risk of underestimated malignancy and the possible morbidity caused by pancreatic surgery.

\section{Potential causes of bias in current literature}

We fear that currently available data on MCN might strongly be biased. Currently, almost exclusively tumors of patients who were considered fit for surgery are 
included. The decision to offer and to accept surgery is always influenced by available preoperative diagnostic findings, the surgeons' interpretation and the patients' characteristics. If, for example, guidelines on pancreatic ductal adenocarcinoma were also solely based on collectives of resected patients, our approach to this disease would surely differ from current treatment standards.

The increasing availability of multiplanar imaging in the last decades, lead to more frequent incidental discovery of cystic pancreatic lesions $(37,38)$. This is accompanied by advances in pancreatic surgery, which allow high-volume centers to safely perform procedures on patients previously deemed unfit surgery (39). One can only speculate if these developments already influenced the reported data on MCN and how it will affect the results of future retrospective studies. Current guidelines, especially those published by the European Study Group, emphasize a tumor size threshold of currently $4 \mathrm{~cm}$ for recommending surgery in asymptomatic patients (3). Our findings did not show a significant association between tumor size and malignancy and available evidence is still limited. We therefore suspect that this approach might be misleading. Symptomatic cysts are generally considered an indication for surgery. However, evaluation of symptoms is dependent on the attending clinician. Given the potential risk of malignant progression in $\mathrm{MCN}$, potentially leading to adenocarcinoma and a significantly worse prognosis, a simple watch-and-wait approach could cause serious harm to individual patients. We therefore recommend that, if feasible, all patients fit for surgery in which the diagnosis of MCN is suspected should at least be offered the attempt of curative resection. If a conservative approach is favored, patient follow up should be performed by experienced pancreatic surgeons in high volume centers.

\section{Conclusions}

Our study found weight loss and elevated levels of CA19-9 to be associated with a higher risk of malignancy in $\mathrm{MCN}$. Current guidelines base indications for surgery mainly on lesions' size and abdominal symptoms. Our patient cohort presented a relatively high percentage of invasive $\mathrm{MCN}$ (25.4\%), including a high proportion of MCN smaller than $4 \mathrm{~cm}$. Based on our findings we feel that the malignant potential of MCN should not be underestimated and a close clinical and radiological follow-up is mandatory in all suspected cases. This is especially important for small lesions, because radiographic changes might indicate malignant transformation well before they surpass $4 \mathrm{~cm}$. Follow-up should be performed by experienced surgeons and radiologists in high volume centers for pancreatic surgery. Surgery should be performed in call cases in which malignancy is suspected, irrespective of the lesion's size.

\section{Acknowledgments}

Funding: None.

\section{Footnote}

Reporting Checklist: The authors have completed the STROBE reporting checklist. Available at http://dx.doi. org/10.21037/atm-20-4774

Data Sharing Statement: Available at http://dx.doi. org/10.21037/atm-20-4774

Peer Review File: Available at http://dx.doi.org/10.21037/ atm-20-4774

Conflicts of Interest: All authors have completed the ICMJE uniform disclosure form (available at http://dx.doi. org/10.21037/atm-20-4774). The authors have no conflicts of interest to declare.

Ethical Statement: The authors are accountable for all aspects of the work in ensuring that questions related to the accuracy or integrity of any part of the work are appropriately investigated and resolved. Written consent was obtained from all patients. The study was approved by the local ethics committee (Nr. 17-6161). The study was conducted in accordance with the Declaration of Helsinki (as revised in 2013).

Open Access Statement: This is an Open Access article distributed in accordance with the Creative Commons Attribution-NonCommercial-NoDerivs 4.0 International License (CC BY-NC-ND 4.0), which permits the noncommercial replication and distribution of the article with the strict proviso that no changes or edits are made and the original work is properly cited (including links to both the formal publication through the relevant DOI and the license). See: https://creativecommons.org/licenses/by-nc-nd/4.0/.

\section{References}

1. Compagno J, Oertel JE. Mucinous cystic neoplasms of the pancreas with overt and latent malignancy 
(cystadenocarcinoma and cystadenoma). A

clinicopathologic study of 41 cases. Am J Clin Pathol 1978;69:573-80.

2. Klöppel G, Solcia E, Longnecker DS, et al. Histological typing of tumours of the exocrine pancreas. Springer Science \& Business Media; 1996.

3. European Study Group on Cystic Tumours of the P. European evidence-based guidelines on pancreatic cystic neoplasms. Gut 2018;67:789-804.

4. Tanaka M, Fernandez-del Castillo C, Adsay V, et al. International consensus guidelines 2012 for the management of IPMN and MCN of the pancreas. Pancreatology 2012;12:183-97.

5. Vege SS, Ziring B, Jain R, et al. American gastroenterological association institute guideline on the diagnosis and management of asymptomatic neoplastic pancreatic cysts. Gastroenterology 2015;148:819-22; quize12-3.

6. Bassi C, Marchegiani G, Dervenis C, et al. The 2016 update of the International Study Group (ISGPS) definition and grading of postoperative pancreatic fistula: 11 Years After. Surgery 2017;161:584-91.

7. Dindo D, Demartines N, Clavien PA. Classification of surgical complications: a new proposal with evaluation in a cohort of 6336 patients and results of a survey. Ann Surg 2004;240:205-13.

8. Sperandei S. Understanding logistic regression analysis. Biochem Med (Zagreb) 2014;24:12-8.

9. Greenland S. Modeling and variable selection in epidemiologic analysis. Am J Public Health 1989;79:340-9.

10. Board WCoTE. WHO Classification of Tumours of the Digestive System. 5th ed. World Health Organization, 2019.

11. Nilsson LN, Keane MG, Shamali A, et al. Nature and management of pancreatic mucinous cystic neoplasm (MCN): A systematic review of the literature. Pancreatology 2016;16:1028-36.

12. Tanaka M, Fernandez-Del Castillo C, Kamisawa T, et al. Revisions of international consensus Fukuoka guidelines for the management of IPMN of the pancreas. Pancreatology 2017;17:738-53.

13. Del Chiaro M, Segersvard R, Pozzi Mucelli R, et al. Comparison of preoperative conference-based diagnosis with histology of cystic tumors of the pancreas. Ann Surg Oncol 2014;21:1539-44.

14. de Pretis N, Mukewar S, Aryal-Khanal A, et al. Pancreatic cysts: Diagnostic accuracy and risk of inappropriate resections. Pancreatology 2017;17:267-72.

15. Procacci C, Carbognin G, Accordini S, et al. CT features of malignant mucinous cystic tumors of the pancreas. Eur Radiol 2001;11:1626-30.

16. Kang CM, Matsushita A, Hwang HK, et al. Experiencebased surgical approach to pancreatic mucinous cystic neoplasms with ovarian-type stroma. Oncol Lett 2018;15:2451-8.

17. Crippa S, Salvia R, Warshaw AL, et al. Mucinous cystic neoplasm of the pancreas is not an aggressive entity: lessons from 163 resected patients. Ann Surg 2008;247:571-9.

18. Thompson LD, Becker RC, Przygodzki RM, et al. Mucinous cystic neoplasm (mucinous cystadenocarcinoma of low-grade malignant potential) of the pancreas: a clinicopathologic study of 130 cases. Am J Surg Pathol 1999;23:1-16.

19. Zamboni G, Scarpa A, Bogina G, et al. Mucinous cystic tumors of the pancreas: clinicopathological features, prognosis, and relationship to other mucinous cystic tumors. Am J Surg Pathol 1999;23:410-22.

20. Kosmahl M, Pauser U, Peters K, et al. Cystic neoplasms of the pancreas and tumor-like lesions with cystic features: a review of 418 cases and a classification proposal. Virchows Arch 2004;445:168-78.

21. Baker ML, Seeley ES, Pai R, et al. Invasive mucinous cystic neoplasms of the pancreas. Exp Mol Pathol 2012;93:345-9.

22. Chang YT, Tien YW, Jeng YM, et al. Overweight increases the risk of malignancy in patients with pancreatic mucinous cystic neoplasms. Medicine (Baltimore) 2015;94:e797.

23. Lee CJ, Scheiman J, Anderson MA, et al. Risk of malignancy in resected cystic tumors of the pancreas $<$ or $=3 \mathrm{~cm}$ in size: is it safe to observe asymptomatic patients? A multi-institutional report. J Gastrointest Surg 2008;12:234-42.

24. Keane MG, Shamali A, Nilsson LN, et al. Risk of malignancy in resected pancreatic mucinous cystic neoplasms. Br J Surg 2018;105:439-46.

25. Martin L, Senesse P, Gioulbasanis I, et al. Diagnostic criteria for the classification of cancer-associated weight loss. J Clin Oncol 2015;33:90-9.

26. Scara S, Bottoni P, Scatena R. CA19-9: Biochemical and Clinical Aspects. Adv Exp Med Biol 2015;867:247-60.

27. Park JW, Jang JY, Kang MJ, et al. Mucinous cystic neoplasm of the pancreas: is surgical resection recommended for all surgically fit patients? Pancreatology 2014;14:131-6.

28. Postlewait LM, Ethun CG, McInnis MR, et al. Association of Preoperative Risk Factors With Malignancy in Pancreatic Mucinous Cystic Neoplasms: A Multicenter 
Study. JAMA Surg 2017;152:19-25.

29. Luo G, Liu C, Guo M, et al. Potential Biomarkers in Lewis Negative Patients With Pancreatic Cancer. Ann Surg 2017;265:800-5.

30. Reddy R, Smyrk T, Zapiach M, et al. Pancreatic mucinous cystic neoplasm defined by ovarian stroma: Demographics, clinical features, and prevalence of cancer. Clin Gastroenterol Hepatol 2004;2:1026-31.

31. Izeradjene $K$, Combs $C$, Best $M$, et al. Kras(G12D) and Smad4/Dpc4 haploinsufficiency cooperate to induce mucinous cystic neoplasms and invasive adenocarcinoma of the pancreas. Cancer Cell 2007;11:229-43.

32. Suzuki Y, Atomi Y, Sugiyama M, et al. Cystic neoplasm of the pancreas: a Japanese multiinstitutional study of intraductal papillary mucinous tumor and mucinous cystic tumor. Pancreas 2004;28:241-6.

33. Yamao K, Yanagisawa A, Takahashi K, et al. Clinicopathological features and prognosis of mucinous cystic neoplasm with ovarian-type stroma: a multiinstitutional study of the Japan pancreas society. Pancreas 2011;40:67-71.

34. Lewis GH, Wang H, Bellizzi AM, et al. Prognosis of minimally invasive carcinoma arising in mucinous

Cite this article as: Höhn P, Soydemir MA, Luu AM, Janot-Matuschek M, Tannapfel A, Uhl W, Belyaev O. It's not all about the size-characteristics and risk factors for malignancy of mucinous cystic neoplasms of the pancreas. Ann Transl Med 2020;8(23):1572. doi: 10.21037/atm-20-4774 cystic neoplasms of the pancreas. Am J Surg Pathol 2013;37:601-5.

35. Sarr MG, Carpenter HA, Prabhakar LP, et al. Clinical and pathologic correlation of 84 mucinous cystic neoplasms of the pancreas: can one reliably differentiate benign from malignant (or premalignant) neoplasms? Ann Surg 2000;231:205-12.

36. Crippa S, Fernandez-Del Castillo C, Salvia R, et al. Mucin-producing neoplasms of the pancreas: an analysis of distinguishing clinical and epidemiologic characteristics. Clin Gastroenterol Hepatol 2010;8:213-9.

37. Ginde AA, Foianini A, Renner DM, et al. Availability and quality of computed tomography and magnetic resonance imaging equipment in U.S. emergency departments. Acad Emerg Med 2008;15:780-3.

38. Kromrey ML, Bulow R, Hubner J, et al. Prospective study on the incidence, prevalence and 5-year pancreatic-related mortality of pancreatic cysts in a population-based study. Gut 2018;67:138-45.

39. Ahola R, Sand J, Laukkarinen J. Centralization of Pancreatic Surgery Improves Results: Review. Scand J Surg 2020;109:4-10. 